\title{
Posterior Dynamic Stabilization with Limited Rediscectomy for Recurrent Lumbar Disc Herniation
}

\author{
Lei Luo, ${ }^{1}$ Chen Zhao, ${ }^{1}$ Pei Li $\mathbb{D}^{1},{ }^{1}$ Liehua Liu, ${ }^{1}$ Qiang Zhou $\left(D,{ }^{1}\right.$ Fei Luo, ${ }^{2}$ and Lichuan Liang ${ }^{1}$ \\ ${ }^{1}$ Department of Orthopedics, The Third Affiliated Hospital of Chongqing Medical University, Chongqing 401120, China \\ ${ }^{2}$ Department of Orthopedics, Southwest Hospital, Army Medical University, Chongqing 400038, China \\ Correspondence should be addressed to Qiang Zhou; zhouqiang@hospital.cqmu.edu.cn
}

Received 5 September 2021; Revised 11 December 2021; Accepted 13 December 2021; Published 23 December 2021

Academic Editor: Sidong Yang

Copyright $(2021$ Lei Luo et al. This is an open access article distributed under the Creative Commons Attribution License, which permits unrestricted use, distribution, and reproduction in any medium, provided the original work is properly cited.

Objective. Recurrent lumbar disc herniation (RLDH) is the most common cause of sciatica after primary discectomy. The purpose of this study was to evaluate the efficacy of transpedicular dynamic stabilization (TDS) combined with limited rediscectomy in the treatment of single-level RLDH. Methods. We retrospectively evaluated a consecutive series of 24 middle-aged patients who underwent TDS (Dynesys system) combined with limited rediscectomy (i.e., removing only extruded or loose disc fragments) for single-level Carragee type II and type IV RLDH between April 2012 and September 2017. Clinical results were evaluated with visual analog scale (VAS) for leg and low back pain, Oswestry Disability Index (ODI) scores, and complications. Imaging data include lumbar segment motion and intervertebral height. Results. The mean follow-up period was 38 months. The VAS and ODI scores were significantly improved at the last follow-up. The average range of motion (ROM) at the stabilized segment was $6.4^{\circ}$ before surgery and $4.2^{\circ}$ at the last follow-up, with a $78.6 \%$ mean preservation $(P<0.05)$. Intervertebral height at the stabilized segment decreased slightly after surgery $(P<0.05)$. However, there was no further decline at the last follow-up. There were no cases of reherniation, screw loosening, or segmental instability. Conclusions. TDS combined with limited rediscectomy resulted in an effective procedure in middle-aged patients with Carragee type II and type IV RLDH. It was able to stabilize the operated segment with partial motion preservation. Moreover, it could maintain disc height and decrease the risk of recurrence in patients with a large posterior annular defect.

\section{Introduction}

Discectomy is considered as the main surgical method in patients with lumbar disc herniation (LDH) without segmental instability [1]. Nevertheless, recurrent lumbar disc herniation $(\mathrm{RLDH})$ is one of the most common complications that can cause severe pain and reoperation after primary discectomy, with reported incidence ranging from $7 \%$ to $24 \%$ [2]. The definition of RLDH has varied among different authors. In most studies, recurrent lumbar disc herniation was defined as a disc hernia at the same level of a previous discectomy in patients with a pain-free interval of at least 6 months long after surgery, regardless of ipsilateral or contralateral herniation [3]. Numerous factors have been associated with an increased rate of reherniation following primary discectomy. Lumbar instability and increased stress upon the intervertebral disc after discectomy may be the major controllable risk factors for RLDH [4].

Surgical treatment for RLDH is indicated in patients with continuous and severe pain, resistant to conservative treatment or cases with motor deficiency. Traditional surgical options include revision discectomy or discectomy supplemented with instrumented fusion. According to the reports by Buchmann et al. [5] and Hou et al. [6], rediscectomy provided good to excellent pain relief in 55\%-96\% of the patients. Because of peridural adhesion, extension interlaminar fenestration would be necessary to reduce the risk of dural and nerve root injury. However, excessive laminectomy may result in lumbar instability. In addition, the intervertebral height decreases after rediscectomy, which can lead to the progression of disc degeneration [7]. Therefore, some surgeons advocate the routine use of 
instrumented fusion in the treatment of RLDH [8]. Intervertebral fusion can maintain lumbar stability and restore intervertebral height. A recent review by Dower et al. [9] demonstrated that fusion resulted in a statistically significant improvement in back pain compared with discectomy (60.1\% vs. $47.2 \%$, respectively). Nonetheless, intervertebral fusion sacrifices the activity of the fixed segment and may accelerate degeneration of adjacent segments [10].

Based on the above deficiencies, transpedicular dynamic stabilization (TDS) was introduced as an alternative to fusion to preserve the activity of the instrumented segments while stabilizing the lumbar spine. Moreover, this technique is targeted to maintain the intervertebral height and reduce the mechanical load upon the disc. It is reported that dynamic stabilization is useful to prevent or diminish recurrent disc herniation after discectomy [11]. Therefore, we proposed TDS combined with limited rediscectomy (i.e., removing only extruded or loose disc fragments) [12] for the treatment of patients with RLDH as an alternative to instrumented fusion. The purpose of this study is to assess the clinical outcomes of TDS with the Dynesys system (Zimmer, USA) for RLDH.

\section{Materials and Methods}

2.1. Patients. This retrospective study included 24 consecutive patients who underwent Dynesys stabilization combined with limited rediscectomy for single-level RLDH from April 2012 to September 2017. There were 16 men and 8 women with an average age of 47.6 years (range of 32-62 years). This study has been approved by the Ethical Committee of the Third Affiliated Hospital of Chongqing Medical University (SKYW20190316). The study was conducted per the ethical principles that have their origins in the Declaration of Helsinki and its subsequent amendments. Informed consent was obtained from all patients. Inclusion criteria were as follows: (1) age $\geq 30$ years at the time of surgery; (2) patients with symptoms of new onset low back pain and radicular leg pain and/or decreased muscular strength and/or abnormal sensation; (3) diagnosis of singlelevel RLDH confirmed by MRI (primary surgery included open discectomy or microendoscopic discectomy); (4) Carragee type II (presence of extruded or sequestered fragments with wide annular rupture; rupture $>6 \mathrm{~mm}$ ) or type IV herniation (the width of the basilar part of the herniated disc $>6 \mathrm{~mm}$; annulus is intact and without free fragments under the annulus) [13]; (5) failed in at least 6 weeks of conservative treatment (oral medication, physical therapy, and so on); and (6) underwent the operation of TDS (Dynesys, Zimmer Spine) combined with limited rediscectomy. Exclusion criteria included the following: (1) more than $1 / 2$ reduction in intervertebral height; (2) rigid segmental kyphosis; and (3) osteoporosis (T-score $\leq-2.5$, DEXA).

2.2. Surgical Procedure. The Dynesys system is a pedicle screw-based dynamic stabilization system. In the system, titanium alloy pedicle screws are connected by a polyethylene terephthalate (PET) cord that goes through the center of a hollow cylinder polycarbonate urethane (PCU) spacer instead of the traditional rigid rod (Figure 1). By appropriately tightening the cord and selecting the length of the spacer, dynamic stabilization would be achieved in the instrumented segment [14].

Patients were placed in the prone position under general anesthesia. A midline dorsal incision in the skin, subcutaneous tissue, and lumbodorsal fascia was applied. Extended interlaminar fenestration decompression was performed through the posterior median approach on the symptomatic side. Only extruded or loose disc fragments were removed. Pedicle screws were inserted through the Wiltse approach under imaging control. The entry point was located at the junction of the lateral border of the superior articular process and the basilar part of the transverse process. Then, the patients' position was modified to obtain the appropriate lumbar lordosis. The spacer was cut according to the measured distance between the screws (distraction force of $1.5 \mathrm{~N}$ ). The central cord and the spacer were then locked within the screw heads. Patients received a soft support lumbar corset for 3 weeks after surgery.

2.3. Clinical and Radiologic Evaluation. Clinical outcomes were assessed with visual analog scale (VAS) for low back and leg pain and Oswestry Disability Index (ODI). Operative time, blood loss, and complications were also documented. Standing plain and dynamic radiographs with flexion and extension views were obtained preoperatively, at 3 months postoperatively, and the last follow-up. The evaluation index included the lordosis at the instrumented segment, the height of the intervertebral disc, and range of motion (ROM) at the instrumented level, the $1^{\text {st }}$ proximal segment, and the lumbar spine (L1-S1). Disc height (DH) was calculated using the anterior intervertebral space height $(\mathrm{AH})$ and posterior intervertebral space height $(\mathrm{PH})$ on the lateral standing lumbar X-ray: $(\mathrm{AH}+\mathrm{PH}) / 2$. Segmental ROM was calculated as the angle difference value between the inferior surface of the upper vertebrae and the superior surface of the lower vertebrae on the lateral standing lumbar flexion-extension $\mathrm{X}$-ray. Disc degeneration grade was evaluated according to the Pfirrmann classification on T2-weighted sagittal and axial MRI [15].

2.4. Statistical Analysis. Statistical analysis was conducted using SPSS (version 16.0, SPSS Inc.). The clinical and radiologic results were analyzed using two-way ANOVA. Significance was defined as $P<0.05$.

\section{Results}

3.1. Perioperative Data and Complications. The mean interval between the primary and revision surgeries was $66.0 \pm 53.2$ months (range of 6-192 months). The RLDH level was L4/5 in 14 (58.3\%) and L5/S1 in 10 (41.7\%) patients (Table 1). The mean operative time was 136 minutes (range 


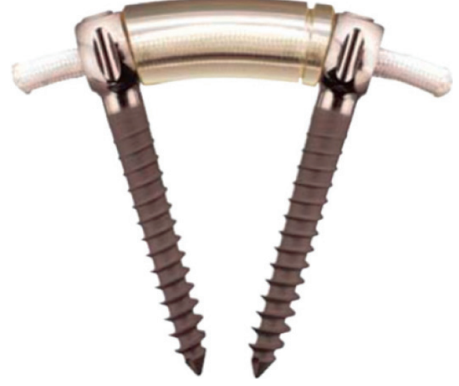

Figure 1: The Dynesys system consists of titanium alloy screws, PET cords, and PCU spacers.

of 98-183 minutes), with an average blood loss of $266 \mathrm{ml}$ (range of $100-500 \mathrm{ml}$ ). The mean follow-up duration was 38 months (range of 28-63 months). Superficial incision infection was observed in one patient 6 days after surgery. The patient was cured by debridement and antibiotics. One patient developed low back and hip pain 3 weeks after surgery, which was relieved after 10 days of conservative treatment. There were no cases of reherniation, screw loosening, or dural and nerve root injury.

3.2. Clinical Outcome. The mean VAS scores for low back pain decreased from $3.8 \pm 0.8$ (range of $3-5$ ) preoperatively to $1.3 \pm 0.6$ (range of $0-2$ ) at 3 months postoperatively and $0.9 \pm 0.4$ (range of $0-2$ ) at the last follow-up. The VAS scores for low back pain were significantly improved at the final follow-up evaluation compared with the baseline values $(P<0.05)$. Similar to the VAS scores for low back pain, the mean VAS scores for leg pain decreased from $5.5 \pm 1.1$ (range of $4-8$ ) to $0.9 \pm 0.6$ (range of $0-2$ ) at 3 months postoperatively and $0.7 \pm 0.5$ (range of $0-1, P<0.05$ ) at the last follow-up. The mean ODI was $57.9 \% \pm 10.6 \%$ (range of $40 \%-76 \%$ ) preoperatively, $23.2 \% \pm 7.8 \%$ (range of $6 \%-$ $40 \%$ ) at the 3 -month follow-up, and $12.8 \% \pm 6.2 \%$ (range of $0 \%-24 \%$ ) at the last follow-up. The changes in $\mathrm{VAS}_{\text {leg }}$ and ODI scores between the preoperative period and the follow-ups were statistically significant as well $(P<0.05)$ (Table 2).

3.3. Radiologic Outcome. The lordosis at instrumented segment was reduced from $8.0^{\circ}$ (range of $-5.7^{\circ}$ to $-13.3^{\circ}$ ) before surgery to $6.8^{\circ}$ (range of $2.3^{\circ}-10.8^{\circ}$ ) at the 3 months follow-up $(P<0.05)$ and $7.0^{\circ}$ (range of $\left.2.6^{\circ}-11.3^{\circ}\right)$ at last follow-up $(P>0.05)$. The average disc height decreased slightly from preoperative $10.4 \mathrm{~mm}$ (range of $6.8 \mathrm{~mm}-13.8 \mathrm{~mm}$ ) to $9.3 \mathrm{~mm}$ (range of $6.3 \mathrm{~mm}-12.6 \mathrm{~mm}$ ) at 3 months postoperatively $(P<0.05)$. There was no further decline at the last follow-up $(P>0.05)$. The average ROM at instrumented segment was $6.4^{\circ}$ (range of $3.1^{\circ}-17.3^{\circ}$ ) before surgery, $4.0^{\circ}$ (range of $2.6^{\circ}-5.9^{\circ}$ ) at 3 months after surgery, and $4.2^{\circ}$ (range of $3.0^{\circ}-5.2^{\circ}$ ) at last follow-up. Compared with preoperatively, $78.6 \%$ (range of $24 \%-152 \%$ ) of ROM was preserved at the last follow-up. The ROM at the $1^{\text {st }}$ proximal segment was $9.0^{\circ}$ (range of $3.5^{\circ}-16.0^{\circ}$ ) before surgery, $9.5^{\circ}$ (range of $5.8^{\circ}-15.7^{\circ}$ ) at 3 months after surgery, and $9.9^{\circ}$ (range of $5.5^{\circ}-14.9^{\circ}$ ) at the last follow-up. The differences were not statistically significant $(P>0.05)$. The lumbar motion was reduced from $34.1^{\circ}$ (range of $13.2^{\circ}-60.4^{\circ}$ ) before surgery to $28.8^{\circ}$ (range of $17.2^{\circ}-40.4^{\circ}$ ) at 3 months after surgery $(P<0.05)$ and $34.8^{\circ}$ (range of $18.7^{\circ}-63.2^{\circ}$ ) at last follow-up $(P>0.05)$ (Table 3$)$. MRI was performed in 11 patients during the follow-up period. Of the 11 patients, the disc degeneration grade (Pfirrmann classification) improved at the index level was observed in 6 patients (Figure 2). The other 5 patients demonstrated no visible signal intensity change at the index level. No progressive degeneration was noted at the first proximal segment in the 11 patients.

\section{Discussion}

The optimal treatment for RLDH remains controversial. Several studies demonstrated that rediscectomy could be able to achieve satisfactory clinical results $[3,6,16]$. However, the chance of segmental lumbar instability increases as rediscectomy often requires more aggressive laminectomy and facetectomy for better exposure of the nerve root canal $[17,18]$. Moreover, excessive sagittal activity at the involved segment after discectomy is a risk factor for recurrent lumbar disc herniation [19, 20]. Furthermore, large posterior annular defect is prone to develop recurrent disc herniation. According to the study by Carragee et al., the recurrence rate after discectomy in patients with Carragee type II and IV herniations was $27 \%$ and $38 \%$, respectively [13]. Discectomy alone might be insufficient to achieve satisfactory results. Thus, we performed posterior dynamic stabilization with limited rediscectomy for the treatment of Carragee type II and type IV RLDH to stabilize the lumbar spine, reduce excessive intervertebral motion, and decrease the risk of re-recurrent disc herniation.

The Dynesys system is supposed to stabilize the operated segment with partial motion preservation. The biomechanical analysis demonstrated that the Dynesys system reduced the intersegmental motion in flexion, extension, lateral bending, and axial rotation compared with structurally damaged specimens so that it could provide substantial stability in case of lumbar degenerative disease $[21,22]$. We investigated the clinical and radiologic results of patients undergoing TDS and limited rediscectomy for RLDH. In general, patients had clinically and statistically significant improvements in $\mathrm{VAS}_{\text {back,leg }}$ and ODI scores. In addition, flexion/extension radiographs showed significant preservation of $\mathrm{ROM}$ at the stabilized segment without lumbar instability or spondylolisthesis. Some studies have also shown that the Dynesys system provides the lumbar spine with sufficient stability in treating degenerative spondylolisthesis $[23,24]$. In the long term, there is always a concern of screw loosening in patients treated with dynamic stabilization [25] although the loosened screws appeared to be asymptomatic [26]. In the present study, no cases of screw loosening were found at the last follow-up. The reasons 
TABle 1: Demographics of dynamic stabilization for recurrent lumbar disc herniation.

\begin{tabular}{|c|c|c|c|c|c|c|c|}
\hline Case no. & Sex & Age (years) & Level & Primary surgery & Recurrence time (months) & Follow-up (months) & Complication \\
\hline 1 & $\mathrm{~F}$ & 53 & L5-S1 & $\mathrm{OD}$ & 120 & 28 & \\
\hline 2 & $\mathrm{~F}$ & 62 & L4-5 & OD & 12 & 49 & \\
\hline 3 & M & 45 & L5-S1 & OD & 60 & 54 & \\
\hline 4 & M & 34 & L4-5 & MED & 19 & 33 & Superficial wound infection \\
\hline 5 & M & 41 & L4-5 & OD & 24 & 42 & \\
\hline 6 & M & 48 & L5-S1 & OD & 120 & 30 & \\
\hline 7 & $\mathrm{~F}$ & 48 & L5-S1 & MED & 9 & 32 & \\
\hline 8 & $\mathrm{~F}$ & 40 & L4-5 & OD & 84 & 30 & \\
\hline 9 & M & 35 & L5-S1 & MED & 36 & 63 & \\
\hline 10 & $\mathrm{M}$ & 58 & L4-5 & OD & 108 & 40 & \\
\hline 11 & $\mathrm{~F}$ & 34 & L4-5 & OD & 60 & 37 & \\
\hline 12 & $\mathrm{M}$ & 53 & L4-5 & OD & 96 & 36 & \\
\hline 13 & $\mathrm{M}$ & 52 & L4-5 & OD & 24 & 46 & \\
\hline 14 & $\mathrm{M}$ & 47 & L4-5 & OD & 6 & 32 & \\
\hline 15 & $\mathrm{~F}$ & 50 & L5-S1 & OD & 192 & 56 & \\
\hline 16 & $\mathrm{~F}$ & 61 & L4-5 & OD & 168 & 30 & \\
\hline 17 & M & 59 & L4-5 & OD & 60 & 47 & \\
\hline 18 & $\mathrm{~F}$ & 58 & L4-5 & OD & 96 & 38 & \\
\hline 19 & $\mathrm{M}$ & 46 & L4-5 & MED & 132 & 34 & Transient low back and hip pain \\
\hline 20 & $\mathrm{~F}$ & 52 & L5-S1 & OD & 7 & 32 & \\
\hline 21 & M & 38 & L5-S1 & OD & 72 & 33 & \\
\hline 22 & M & 43 & L5-S1 & OD & 24 & 29 & \\
\hline 23 & M & 54 & L5-S1 & OD & 24 & 33 & \\
\hline 24 & M & 32 & L4-5 & OD & 30 & 36 & \\
\hline
\end{tabular}

M: male, F: female, OD: open discectomy, and MED: microendoscopic discectomy.

might be the patients with osteoporosis were excluded, and minimal bone resection as well as pedicle screws placement lateral to the facet joints that makes the rotation axis of the Dynesys system close to the rotation axis of the motion segment could reduce the stress on the system.

Disc removal may lead to accelerated disc degeneration at the operative level. Disc height reduction and endplate degeneration may be the most common findings following discectomy [27, 28]. Excessive removal is associated with the progression of disc space narrowing, which may lead to low back pain over time [29]. Conversely, limited nucleus pulposus removal, preserved disc height, and moderate disk degeneration are significant risk factors for RLDH $[19,30,31]$. Therefore, surgical treatment which can both preserve the disc height and decrease the incidence of reherniation may allow for improved outcomes. According to the literature, discectomy with additional transpedicular dynamic stabilization is useful to prevent progression of intervertebral disc degeneration and decrease the risk of recurrence in treating primary lumbar disc herniation $[32,33]$. Our results showed that disc height decreased slightly after TDS and limited discectomy, but there was no further decline at the last follow-up. The disc height could be maintained at the last follow-up compared with the postoperative value. The result may indicate that dynamic stabilization can delay or prevent the progression of disc degeneration. Of the 11 patients who underwent MRI examination during the follow-up period, 6 patients demonstrated improved disc degeneration grade at the index level, whereas the other patients showed no visible signal intensity change at the index level. In addition, a second recurrence
TABle 2: Clinical outcomes.

\begin{tabular}{lccccc}
\hline & Preoperative & 3 months & $\begin{array}{c}\text { Last } \\
\text { follow-up }\end{array}$ & $F$ & $P$ \\
\hline $\mathrm{VAS}_{\text {back }}$ & $3.8 \pm 0.8$ & $1.3 \pm 0.6$ & $0.9 \pm 0.4$ & 110.49 & 0.001 \\
$\mathrm{VAS}_{\text {leg }}$ & $5.5 \pm 1.1$ & $0.9 \pm 0.6$ & $0.7 \pm 0.5$ & 525.16 & 0.001 \\
ODI (\%) & $57.9 \pm 10.6$ & $23.2 \pm 7.8$ & $12.8 \pm 6.2$ & 171.475 & 0.001 \\
\hline
\end{tabular}

did not occur in any patients in this study at the last followup. This further confirms that the Dynesys system can decelerate the degeneration process. However, its mechanism remains unclear. One possible mechanism is that intradiscal pressure is reduced by axial distraction [34], and moderate dynamic compression or distraction could promote anabolism in nucleus pulposus cells $[35,36]$, thus allowing the disc to repair itself. Some studies reported disc rehydration at the bridged level after dynamic stabilization [37, 38]. However, severe degeneration of the disc is difficult to reverse. Therefore, patients with a significant reduction in intervertebral disc height were excluded from this study.

There were limitations to this study: lack of a control group, limited patient number, and short follow-up period. Nevertheless, these are common issues when evaluating a new surgical technique. Besides, there was no further analysis of the causes of low back pain before the second surgery and the role of dynamic stabilization in alleviating it. In addition, due to the high costs, only 11 patients received MRI at postoperative follow-up in the study. A longer follow-up period and more patients receiving MRI will contribute to better observation of the disc changes after dynamic stabilization. 
TABLE 3: Radiographic outcomes.

\begin{tabular}{|c|c|c|c|c|c|}
\hline & Preoperative & 3 months & Last follow-up & $F$ & $P$ \\
\hline Lordosis at instrumented segment $\left({ }^{\circ}\right)$ & $8.0 \pm 4.2$ & $6.8 \pm 2.4$ & $7.0 \pm 2.6$ & 2.379 & 0.104 \\
\hline Disc height at instrumented segment (mm) & $10.4 \pm 1.9$ & $9.3 \pm 1.9$ & $9.1 \pm 1.8$ & 57.562 & 0.001 \\
\hline ROM at instrumented segment $\left({ }^{\circ}\right)$ & $6.4 \pm 3.2$ & $4.0 \pm 0.9$ & $4.2 \pm 0.6$ & 12.578 & 0.001 \\
\hline ROM at the $1^{\text {st }}$ proximal segment $\left({ }^{\circ}\right)$ & $9.0 \pm 3.9$ & $9.5 \pm 2.8$ & $9.9 \pm 2.6$ & 1.969 & 0.151 \\
\hline $\mathrm{ROM}$ at L1-S1 $\left({ }^{\circ}\right)$ & $34.1 \pm 13.1$ & $29.2 \pm 6.8$ & $34.8 \pm 10.5$ & 4.496 & 0.016 \\
\hline
\end{tabular}

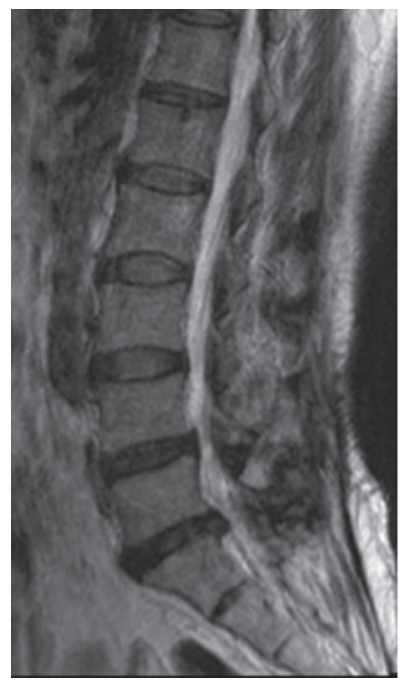

(a)

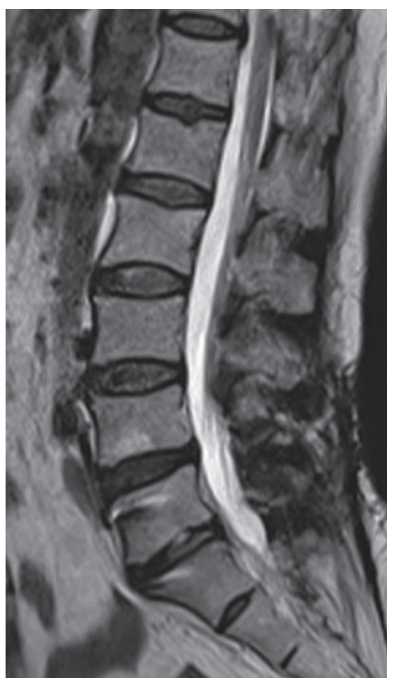

(b)

Figure 2: A case of a 53 years old female patient with RLDH at L5-S1. MRI scans showed rehydration at 22-month follow-up after transpedicular dynamic stabilization.

\section{Conclusions}

Our results suggest that TDS combined with limited rediscectomy resulted in a safe and effective procedure in middle-aged patients with Carragee type II and type IV RLDH. It was able to stabilize the operated segment with partial motion preservation. Moreover, it could maintain disc height and decrease the risk of recurrence in patients with a large posterior annular defect.

\section{Data Availability}

The data used to support the findings of this study are available from the corresponding author upon request.

\section{Conflicts of Interest}

The authors declare that there are no conflicts of interest regarding the publication of this paper.

\section{Authors' Contributions}

LL and QZ conceived and designed the study. QZ and FL carried out the operation. LL, CZ, PL, and LC L (Lichuan Liang) collected the data. LH L (Liehua Liu) analyzed the data. LL and QZ wrote the manuscript. All authors have read and approved the final manuscript.

\section{Acknowledgments}

This study was supported by Postdoctoral Research Start-Up Fund of Chongqing Medical University (R9005).

\section{References}

[1] A. Blamoutier, "Surgical discectomy for lumbar disc herniation: surgical techniques," Orthopaedics \& Traumatology, Surgery \& Research: OTSR, vol. 99, no. 1 Suppl, pp. S187-S196, 2013.

[2] J. A. O’Donnell, J. T. Anderson, A. R. Haas et al., "Treatment of recurrent lumbar disc herniation with or without fusion in workers' compensation subjects," Spine (Phila. Pa. 1976), vol. 42, no. 14, pp. E864-E870, 2017.

[3] H. Mashhadinezhad, E. Sarabi, S. Mashhadinezhad, and B. Ganjeifar, "Clinical outcomes after microdiscectomy for recurrent lumbar disk herniation: a single-center study," The archives of bone and joint surgery, vol. 6, no. 5, pp. 397-401, 2018.

[4] C. U. Onyia and S. K. Menon, "Impact of comorbidities on outcome following revision of recurrent single-level lumbar disc prolapse between revision microdiscectomy and posterior lumbar interbody fusion: a single-institutional analysis," Asian Journal of Neurosurgery, vol. 14, no. 2, pp. 392-398, 2019.

[5] N. Buchmann, A. Preuß, J. Gempt et al., "Outcome after surgical treatment for late recurrent lumbar disc herniations in standard open microsurgery," World Neurosurgery, vol. 89, pp. 382-386, 2016. 
[6] T. Hou, Q. Zhou, F. Dai et al., "Repeated microendoscopic discectomy for recurrent lumbar disk herniation," Clinics, vol. 70, no. 2, pp. 120-125, 2015.

[7] J. P. Jarman, V. E. Arpinar, D. Baruah, A. P. Klein, D. J. Maiman, and L. Tugan Muftuler, "Intervertebral disc height loss demonstrates the threshold of major pathological changes during degeneration," European Spine Journal, vol. 24, no. 9, pp. 1944-1950, 2015.

[8] T. E. Mroz, D. Lubelski, S. K. Williams et al., "Differences in the surgical treatment of recurrent lumbar disc herniation among spine surgeons in the United States," The Spine Journal, vol. 14, no. 10, pp. 2334-2343, 2014.

[9] A. Dower, R. Chatterji, A. Swart, and M. J. Winder, "Surgical management of recurrent lumbar disc herniation and the role of fusion," Journal of Clinical Neuroscience, vol. 23, pp. 44-50, 2016.

[10] S. Okuda, Y. Nagamoto, T. Matsumoto, T. Sugiura, Y. Takahashi, and M. Iwasaki, “Adjacent segment disease after single segment posterior lumbar interbody fusion for degenerative spondylolisthesis: minimum 10 years follow-up," Spine (Phila. Pa. 1976), vol. 43, no. 23, pp. E1384-E1388, 2018.

[11] A. F. Ozer, F. Keskin, T. Oktenoglu et al., "A novel approach to the surgical treatment of lumbar disc herniations: indications of simple discectomy and posterior transpedicular dynamic stabilization based on carragee classification," Advances in Orthopedics, vol. 2013, Article ID 270565, 6 pages, 2013.

[12] E. J. Carragee, A. O. Spinnickie, T. F. Alamin, and S. Paragioudakis, "A prospective controlled study of limited versus subtotal posterior discectomy: short-term outcomes in patients with herniated lumbar intervertebral discs and large posterior anular defect," Spine (Phila. Pa. 1976), vol. 31, no. 6, pp. 653-657, 2006.

[13] E. J. Carragee, M. Y. Han, P. W. Suen, and D. Kim, "Clinical outcomes after lumbar discectomy for sciatica," Journal of Bone and Joint Surgery American Volume, vol. 85, no. 1, pp. 102-108, 2003.

[14] O. Schwarzenbach and U. Berlemann, "Dynamic posterior stabilization with the pedicle screw system DYNESYS ${ }^{\circledR}, "$ Operative Orthopädie und Traumatologie, vol. 22, no. 5-6, pp. 545-557, 2010.

[15] C. W. A. Pfirrmann, A. Metzdorf, M. Zanetti, J. Hodler, and N. Boos, "Magnetic resonance classification of lumbar intervertebral disc degeneration," Spine (Phila. Pa. 1976), vol. 26, no. 17, pp. 1873-1878, 2001.

[16] C. Mehren, L. Wanke-Jellinek, and A. Korge, "Revision after failed discectomy," European Spine Journal, vol. 29, 2019.

[17] S. Ramhmdani, Y. Xia, R. Xu et al., "Iatrogenic spondylolisthesis following open lumbar laminectomy: case series and review of the literature," World Neurosurgery, vol. 113, pp. e383-e390, 2018.

[18] A. Okawa, K. Shinomiya, K. Takakuda, and O. Nakai, "A cadaveric study on the stability of lumbar segment after partial laminotomy and facetectomy with intact posterior ligaments," Journal of Spinal Disorders, vol. 9, no. 6, pp. 518-526, 1996.

[19] K.-T. Kim, D.-H. Lee, D.-C. Cho, J.-K. Sung, and Y.-B. Kim, "Preoperative risk factors for recurrent lumbar disk herniation in L5-S1," Journal of Spinal Disorders \& Techniques, vol. 28, no. 10, pp. E571-E577, 2015.

[20] E.-H. Shin, K.-J. Cho, Y.-T. Kim, and M.-H. Park, "Risk factors for recurrent lumbar disc herniation after discectomy," International Orthopaedics, vol. 43, no. 4, pp. 963-967, 2019.

[21] W. Schmoelz, J. F. Huber, T. Nydegger, D. Ing, L. Claes, and H. J. Wilke, "Dynamic stabilization of the lumbar spine and its effects on adjacent segments," Spine (Phila. Pa. 1976), vol. 28, no. 4, pp. 418-423, 2003.

[22] C. A. Niosi, Q. A. Zhu, D. C. Wilson, O. Keynan, D. R. Wilson, and T. R. Oxland, "Biomechanical characterization of the three-dimensional kinematic behaviour of the Dynesys dynamic stabilization system: an in vitro study," European Spine Journal, vol. 15, no. 6, pp. 913-922, 2006.

[23] S. W. Yu, S. C. Yang, C. H. Ma, C. H. Wu, C. Y. Yen, and Y. K. Tu, "Comparison of Dynesys posterior stabilization and posterior lumbar interbody fusion for spinal stenosis L4L5," Acta Orthopaedica Belgica, vol. 78, no. 2, pp. 230-239, 2012.

[24] C. H. Kuo, P. Y. Chang, J. C. Wu et al., "Dynamic stabilization for L4-5 spondylolisthesis: comparison with minimally invasive transforaminal lumbar interbody fusion with more than 2 years of follow-up," Neurosurgical Focus, vol. 40, no. 1, pp. E3-E9, 2016.

[25] M. Prud'homme, C. Barrios, P. Rouch, Y. P. Charles, J.-P. Steib, and W. Skalli, "Clinical outcomes and complications after pedicle-anchored dynamic or hybrid lumbar spine stabilization: a systematic literature review," Journal of Spinal Disorders \& Techniques, vol. 28, no. 8, pp. E439-E448, 2015.

[26] J. C. Wu, W. C. Huang, H. W. Tsai et al., "Pedicle screw loosening in dynamic stabilization: incidence, risk, and outcome in 126 patients," Neurosurgical Focus, vol. 31, no. 4, p. E9, 2011.

[27] M. Barth, M. Diepers, C. Weiss, and C. Thomé, "Two-year outcome after lumbar microdiscectomy versus microscopic sequestrectomy: part 2: radiographic evaluation and correlation with clinical outcome," Spine (Phila. Pa. 1976), vol. 33, no. 3, pp. 273-279, 2008.

[28] B. K. Weiner, M. Vilendecic, D. Ledic et al., "Endplate changes following discectomy: natural history and associations between imaging and clinical data," European Spine Journal, vol. 24, no. 11, pp. 2449-2457, 2015.

[29] M. J. DePalma, J. M. Ketchum, T. R. Saullo, and B. L. Laplante, "Is the history of a surgical discectomy related to the source of chronic low back pain?" Pain Physician, vol. 15, no. 1, pp. E53-E58, 2012.

[30] M. J. Mcgirt, S. Eustacchio, P. Varga et al., "A prospective cohort study of close interval computed tomography and magnetic resonance imaging after primary lumbar discectomy," Spine, vol. 34, no. 19, pp. 2044-2051, 2009.

[31] E. Yorimitsu, K. Chiba, Y. Toyama, and K. Hirabayashi, "Long-term outcomes of standard discectomy for lumbar disc herniation: a follow-up study of more than 10 years," Spine (Phila. Pa. 1976), vol. 26, no. 6, pp. 652-657, 2001.

[32] M. Putzier, S. V. Schneider, J. F. Funk, S. W. Tohtz, and C. Perka, "The surgical treatment of the lumbar disc prolapse: nucleotomy with additional transpedicular dynamic stabilization versus nucleotomy alone," Spine (Phila. Pa. 1976), vol. 30, no. 5, pp. 109-114, 2005.

[33] T. Kaner, M. Sasani, T. Oktenoglu, M. Cosar, and A. F. Ozer, "Clinical outcomes after posterior dynamic transpedicular stabilization with limited lumbar discectomy: Carragee classification system for lumbar disc herniations," SAS Journal, vol. 4, no. 3, pp. 92-97, 2010.

[34] C. Schilling, S. Krüger, T. M. Grupp, G. N. Duda, W. Blömer, and A. Rohlmann, "The effect of design parameters of dynamic pedicle screw systems on kinematics and load bearing: an in vitro study," European Spine Journal, vol. 20, no. 2, pp. 297-307, 2011.

[35] P. Li, Y. Gan, H. Wang et al., "Dynamic compression effects on immature nucleus pulposus: a study using a novel intelligent and mechanically active bioreactor," International Journal of Medical Sciences, vol. 13, no. 3, pp. 225-234, 2016. 
[36] M. Kroeber, F. Unglaub, T. Guehring et al., "Effects of controlled dynamic disc distraction on degenerated intervertebral discs: an in vivo study on the rabbit lumbar spine model," Spine (Phila. Pa. 1976), vol. 30, no. 2, pp. 181-187, 2005.

[37] A. Yilmaz, S. Senturk, M. Sasani et al., "Disc rehydration after dynamic stabilization: a report of 59 cases," Asian Spine Journal, vol. 11, no. 3, pp. 348-355, 2017.

[38] L.-Y. Fay, J.-C. Wu, T.-Y. Tsai et al., "Intervertebral disc rehydration after lumbar dynamic stabilization: magnetic resonance image evaluation with a mean followup of four years," Advances in Orthopedics, vol. 2013, Article ID 437570, 8 pages, 2013 